\title{
IN VITRO EVALUATION OF THE ANTIMICROBIAL EFFECT OF ENDOSEQUENCE BC AND AH PLUS ROOT CANAL SEALERS ON ENTEROCCOCUS FAECALIS
}

\author{
Howida A. Enan ${ }^{*}$ BDS, Eman A. Omran ${ }^{2} P h D$, Rania M. El Backly³ PhD, Ashraf M. \\ Zaazou $^{4,5}$ PhD, Mohamed M. Ibrahim ${ }^{5}$ PhD
}

ABSTRACT

INTRODUCTION: Remaining microorganisms after chemo-mechanical cleaning and disinfection may be eliminated or rendered harmless by entombing them through complete obturation with gutta percha points and sealer. Adequate antimicrobial effect is of optimum importance to achieve root canal treatment success.

OBJECTIVES: The aim of this study was to investigate the antimicrobial potential of two types of sealers against Enterococcusfaecalis present in dentinal tubules, over a 30 day period.

MATERIALS AND METHODS: Cells of Enterococcus faecalis were introduced into the dentinal tubules by incubation in brain-heart infusion broth for 3 weeks. AH Plus and Endosequence BioCeramic sealer (BC sealer) were coated on the root canal wall of the dentin specimens and cold lateral obturation technique was preformed, specimens were then stored for one, seven, and 30 days at $37^{\circ} \mathrm{C}$. At the end of each incubation period, dentin powder was collected by sequential removal of dentin by the use of peeso reamers throughout the sample thickness, total bacterial count was assessed by Colony forming units (CFU) count.

RESULTS: No significant difference was observed in the total bacterial count among the three groups in day one. AH Plus showed statistically significant lower bacterial counts in comparison to the positive control group in day 7 and day 30, while Endosequence BC showed a statistically significant lower bacterial count in comparison to the positive control group at day 30.

CONCLUSIONS: AH plus exhibited a stronger antibacterial affect after setting and therefore is more effective than Endosequence BC in eliminating Enterococcus faecalis from the root canal system. In addition, both sealers lose their antibacterial effect with time.

KEYWORDS: Enterococcusfaecalis, Bioceramic, AH Plus, Sealer, Antimicrobial, Penetration.

1. Clinical Instructor at Conservative Dentistry Department, Faculty of Dentistry, Pharos University, Alexandria, Egypt.

2. Lecturer of Microbiology, High Institute of Public Health, Alexandria University, Egypt

3. Associate Professor of Endodontics, Conservative Dentistry Department, Faculty of Dentistry, Alexandria University, Egypt

4. Professor of Endodontics, Department of Endodontics, Faculty of Dentistry, King Abdel Aziz University, KSA.

5. Professor of Endodontics, Conservative Dentistry Department, Faculty of Dentistry, Alexandria University, Egypt.

*Corresponding author howida.enan@gmail.com

\section{INTRODUCTION}

One of the main goals of endodontic treatment is to eliminate microorganisms from the contaminated root canal anatomy. Root canal treatment reduces but does not necessarily dispose of all microbes, and viable bacteria often remain in the dentinal tubules and parallel channels such as the lateral canals. The utilization of root obturation with sealers having antibacterial activity is considered gainful in the effort to further decrease the quantity of residual microorganisms or even destroy the infection completely (1). Thus, analyzing the antibacterial action of root canal sealers against these bacteria is relevant to clinical practice.

Enterococcus faecalis, a gram positive cocci is reported to be the most common microorganism in infected root canals and in re-treatment cases of persistent apical periodontitis. Its prevalence has been found to reach up to $77 \%$ (2). Enterococcus faecalis endure unforgiving environments including extraordinary alkaline $\mathrm{pH}$ 11.1. It was also found to be the most commonly used microorganism in in vitro studies to evaluate the antimicrobial efficiency of root canal sealers related to persistent periapical infections (3).

Several studies have been conducted to investigate the antimicrobial potential of different endodontic sealers. It's an established fact that the epoxy resin-based sealer AH Plus (DENTSPLY DeTrey GmbH, Konstanz, Germany) is the gold standard sealer regarding physical properties; it is hydrophobic exhibiting a high bond strength to dentin when compared to other root canal sealers. AH Plus has been shown to exhibit 
adequate dimensional stability, minimal solubility and disintegration (4). In addition to a high antibacterial effect when freshly mixed and that can be sustained for several days. Endosequence BC Sealer (Brasseler USA, Savannah, GA) is a pre-mixed and injectable bioceramic endodontic sealer, composed of zirconium oxide, $\mathrm{Ca} 2 \mathrm{SiO} 4$, calcium phosphate monobasic, $\mathrm{Ca}(\mathrm{OH}) 2$, filler, and thickening agents. It is hydrophilic and utilizes dentinal tubules' moisture to initiate and finalize its setting reaction. The manufacturer also states that the sealer is highly biocompatible and is antibacterial during its setting reaction because of its highly alkaline $\mathrm{pH}$ $(\geq 12)(5)$.

The in vitro mimicking of bacterial infiltration in dentinal tubules has been examined and improved for many years. Most of the studies with bacterial infiltration model have been founded on cultural methods; allowing bacteria to be grown in media in the root canals of extracted teeth or preprepared dentin blocks. A dentin block model, which has been advocated for the assessment of the antibacterial effect in dentin for more than twenty years, allowed bacterial homing into dentinal tubules for up to $500 \mu \mathrm{m}$ from the main root canal (6).

Thus this study was conducted to assess the antimicrobial ability of two chemically different root canal sealers; Endosequence BC Sealer and AH Plus sealer over a thirty day period in eradicating the bacterial contamination inside the dentinal tubules of the root canal, using an in vitro model contaminated with Enterococcus faecalis. The null hypothesis was that there was no difference between the two tested sealers.

\section{MATERIALS AND METHODS \\ Specimens' selection}

This study was approved by the ethical committee of the faculty of dentistry, Alexandria University (IRB:NO: 00010556-IORG0008839). It was conducted on fifty five mature single rooted freshly extracted teeth for orthodontic or periodontal reasons. Teeth exhibiting internal or external root resorption, previous root canal treatment, root caries and preaccessed were excluded. The teeth were stored in $0.01 \%$ sodium hypochlorite at $4^{\circ} \mathrm{C}$ after cleaning the root surface with a curette.

\section{Specimens' Preparation}

Root segments with a length of seven mm were prepared by apical resection and cutting the crown two-three mm below the cementoenamel junction using rotating diamond disc under water cooling mounted on a low speed handpiece. Their length was confirmed using a digital caliper. Each root canal was enlarged to size two peeso reamer (Mani, Tochigi, Japan) under irrigation with two $\mathrm{ml} 5.25 \%$ sodium hypochlorite in order to form an apical seat of ISO size 90. The outer surfaces of the root specimens were prepared using a high speed extra fine diamond bur under constant water cooling, to achieve a standard thickness of two mm all around the canal diameter and to remove the root surface cementum. Organic and inorganic debris including the smear layer were removed by intracanal irrigation with one $\mathrm{ml}$ of $17 \%$ EDTA for one minute followed by three $\mathrm{ml}$ of $5.25 \% \mathrm{NaOCl}$, each irrigant was activated by Endovac (Kerr Endo, Orange Country, CA). The specimens were then immersed in an ultrasonic bath for extracanal smear layer removal by $17 \%$ EDTA followed by $5.25 \% \mathrm{NaOCl}$ each for four minutes. Specimens were then rinsed individually in sterile water for one minute after smear layer removal. A random specimen (one tooth) was longitudinally sectioned to be observed under the scanning electron microscope, to ensure complete removal of the smear layer (6). (Figure 1)

\section{Specimens' sterilization}

The specimens were then individually placed in test tubes with screw caps containing three $\mathrm{mL}$ of Brain-Heart Infusion Medium (BHI; Oxoid, Basingstoke, UK) and autoclaved for 20 minutes at $121^{\circ} \mathrm{C}$. The specimens were then kept in an incubator at $37^{\circ} \mathrm{C}$ for 24 hours to check the efficacy of the sterilization treatment $(7)$.

To confirm the specimens' sterilization, after the incubation period was over, each tube was mixed using a vortex mixer for 10 seconds, after which $25 \mu \mathrm{L}$ were taken from each test tube and streaked over the surface of a blood agar plate using surface spread method. Incubation was done overnight aerobically at $37^{\circ} \mathrm{C}$. After 24 hours of incubation, all the plates were examined for bacterial growth. No growth was found in all the streaked plates confirming sterilization of the specimens to be used.

\section{Specimens' infection with Enterococcus faecalis}

An Enterococcus faecalis reference strain (ATCC 29212) was grown on a blood agar plate after incubation aerobically at $37^{\circ} \mathrm{C}$ for 24 hours. (Figure 2)

The purity of the culture was confirmed by examining isolated colonies of Enterococcus faecalis macroscopically on blood agar plates. Colonies of Enterococcus faecalis appeared as small, creamy or white, smooth diplococci, round and nonhemolytic. Further confirmation was done through Gram staining, and by the use of MALDI-TOF mass spectrometry (Bruker Daltonics GmbH,Germany) at the Microbiology Department in the Faculty of Medicine, Alexandria University. Confirmed Enterococcus faecalis isolates were suspended in tubes containing Brain-Heart Infusion Medium and were adjusted spectrophotometrically to match the turbidity of $1.5 \times 10^{8}$ colony forming unit (CFU) $\mathrm{mL}^{-1}$ (equivalent to \pm 0.5 McFarland standard). The teeth were then individually placed in glass test tubes with screw caps containing three $\mathrm{mL}$ of the same solution; Enterococcus faecalis inoculated Brain-Heart Infusion Medium (BHI; Oxoid, Basingstoke, UK). The test tubes were closed and kept at $37^{\circ} \mathrm{C}$ for three weeks in an incubator (7).

During the three weeks of incubation, replenishing of the inoculum in each test tube was done twice weekly near an open flame using sterile pipettes. In which the existing broth was alternatively replaced by fresh bacterial suspension or Brain-Heart Infusion broth every other day. Every seven days, random sampling was done for gram staining to confirm the purity and viability of the Enterococcus faecalis cultures (7). 
The fifty four specimens were divided into three main groups with 18 specimens each according to the type of sealer to be used; Group I Endosequence BC Sealer, Group II AH Plus and Group III Positive Control (distilled water and gutta percha). The main Groups were further subdivided into three subgroups according to the time of incubation period of each sealer; Subgroups a , b and c ( The antimicrobial effect was evaluated after one day, seven days and 30 days of incubation respectively).

\section{Specimens' obturation}

After the three week bacterial incubation period, the specimens were removed from the glass test tube and rinsed in sterile water for one minute and air-dried. The outer surfaces (cemental sides) of the specimens were closed by nail varnish. The specimens were then randomly divided into three groups according to the sealer to be used; Group I: Endosequence BC used as the calcium silicate based root canal sealer, Group II: AH Plus used as the epoxy resin root canal sealer, Group III: distilled water served as the positive control. Each sealer was prepared according to each manufacturer's instructions \& cold lateral obturation was performed. (Figure 3)

All the specimens were then incubated at $37^{\circ} \mathrm{C}$ in $100 \%$ relative humidity for one, seven, and thirty days.

Dentin sampling procedure for antimicrobial testing

On completion of each incubation time, the root canal was reestablished by removal of the gutta percha with sterile peeso reamers size two (ISO 0.90). Sterile peeso reamers sizes three, four, five and six (ISO 1.10, 1.30, 1.50 and 1.70) were used to collect dentin powder from the canal with the specimens held in a sterile clamp. To standardize measurements, the dentine powder was weighed using a sensitive analytical balance, to achieve a $0.0045 \mathrm{~g}$ from each specimen (8).

The dentin powder obtained from each specimen was immediately collected in sterile eppendorfs containing $1.5 \mathrm{ml}$ BHI.(Figure 4) The eppendorfs were then vortexed for ten seconds and a sterile micropipette tip was used to collect $25 \mu \mathrm{L}$ that were streaked over the surface of a blood agar plate using surface spread method. Following 24 hours incubation at $37^{\circ} \mathrm{C}$, visible colonies were counted and the total colony-forming unit per $\mathrm{ml}(\mathrm{CFU} / \mathrm{ml})$ was calculated.

Confirmation of the purity and identity Enterococcus faecalis was done through Gram staining, and by the use of MALDI-TOF mass spectrometry at the microbiology department in the faculty of medicine, Alexandria University and statistical analysis was performed on the data obtained.

\section{Statistical analysis}

Data were collected and entered to the computer using SPSS (Statistical Package for Social Science) program for statistical analysis (ver 21) (9). Data were entered as numerical or categorical, as appropriate. Kolmogorov-Smirnov test of normality revealed significance in the distribution of most of the variables, so the non-parametric statistics was adopted (10). Data were described using minimum, maximum, mean, standard deviation and 95\% CI of the mean (11), median and inter-quartile range for not-normally distributed data.Comparisons were carried out between more than two studied independent not-normally distributed subgroups using Kruskal-Wallis test (12). Post-hoc pair-wise comparisons when Kruskal-Wallis test or Friedman test were significant was carried out using Dunn- Sidak test for multiple comparison(13). An alpha level was set to 5\% with a significance level of $95 \%$, and a beta error accepted up to $20 \%$ with a power of study of $80 \%$.

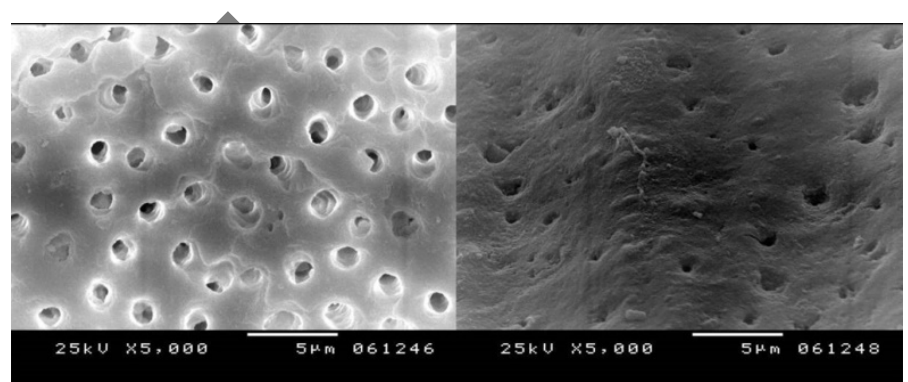

Figure (1): Scanning electron microscope pictures showing complete smear layer removal; a) Intracanal smear layer removed and dentinal tubules open b) External root wall, smear layer removed and dentinal tubules open

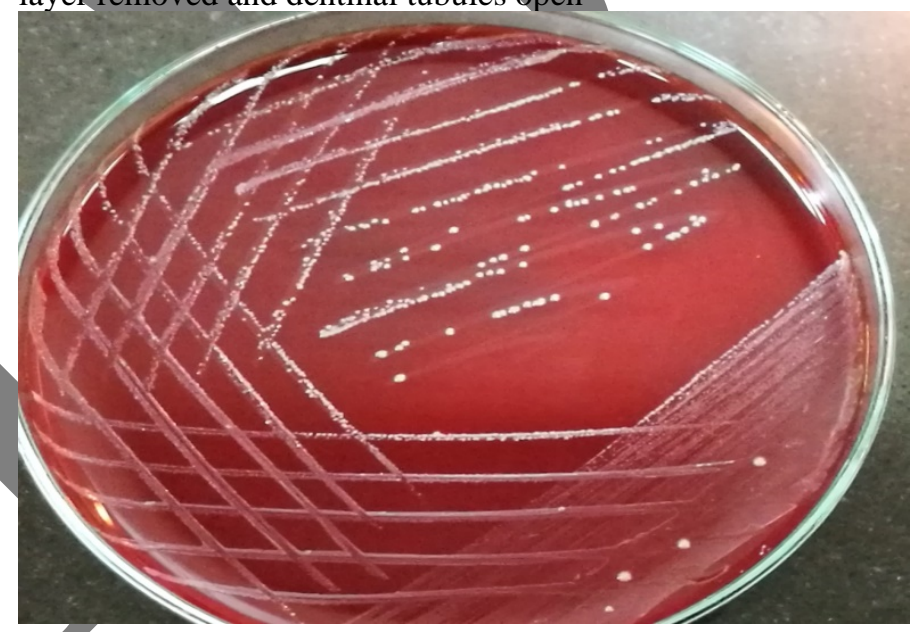

Figure (2): An Enterococcus faecalis reference strain (ATCC 29212)

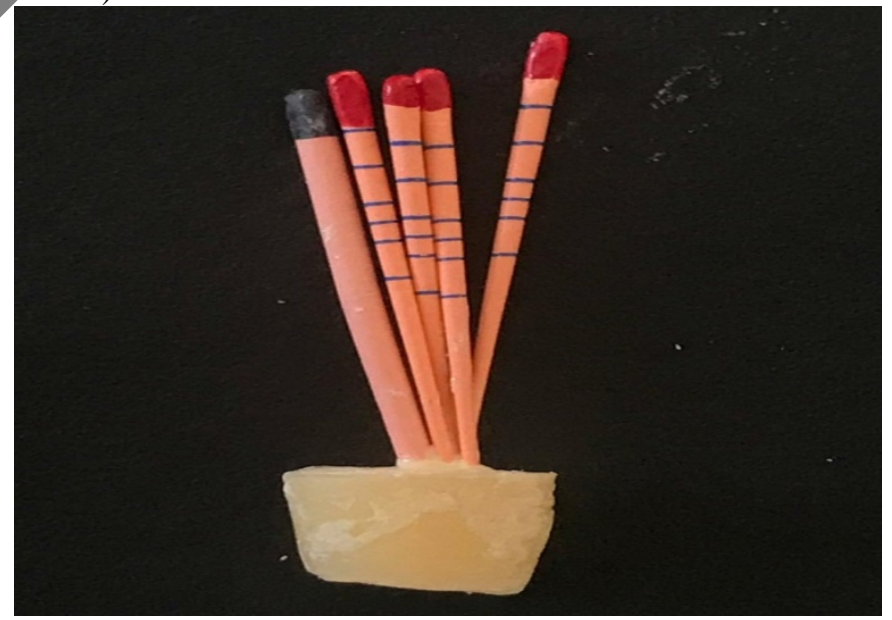

Figure (3): Specimen showing cold lateral obturation. 


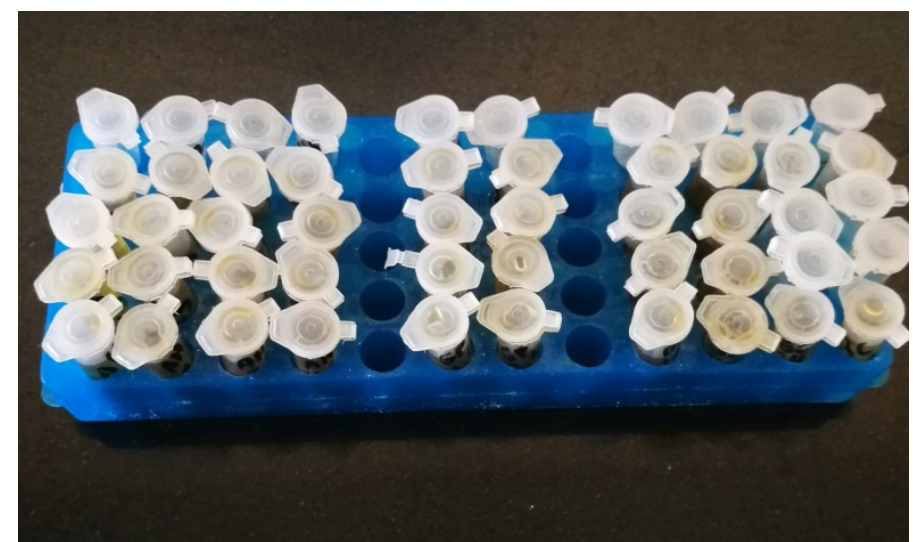

Figure (4) :The dentin powder obtained from each specimen at each ISO size was immediately collected in sterile eppendorfs containing $1.5 \mathrm{ml} \mathrm{BHI}$.

\section{RESULTS}

Regarding the total bacterial count comparison between the three studied groups at day one, Kruskal Wallis test revealed no significant difference in the total bacterial count among the three groups. However at day seven, Kruskal Wallis test showed a significant difference between the three studied groups $(p=0.000)$. Pairwise comparisons revealed a significantly lower total bacterial count in the AH plus root canal sealer group with a median of $1218.75 \mathrm{cfu} / \mathrm{ml}$ compared to a median of $35831.25 \mathrm{cfu} / \mathrm{ml}$ for the positive control group $(\mathrm{p}=0.000)$. Furthermore at day 30, Kruskal Wallis test showed a significant difference between the three studied groups $(\mathrm{p}=$ 0.002). Pairwise comparisons revealed a significantly lower total bacterial count in the $\mathrm{AH}$ plus root canal sealer group with a median of $39487.50 \mathrm{cfu} / \mathrm{ml}$ compared to a median of $242937.50 \mathrm{cfu} / \mathrm{ml}$ in the positive control group $(\mathrm{p}=0.002)$. As well as, a significantly lower total bacterial count in the Endosequence BC root canal sealer group with a median $49887.50 \mathrm{cfu} / \mathrm{ml}$ compared to a median $242937.50 \mathrm{cfu} / \mathrm{ml}$ in the positive control group $(\mathrm{p}=0.048)$. (Table 1) (Figure 5)

Regarding the total bacterial count comparison between the three studied time periods, although there was a bacterial count decrease from day one to day seven in both group I and II it was not of significant difference. In group I, Kruskal Wallis test showed a significant difference between the three studied time periods $(\mathrm{p}=0.014)$. Pairwise comparisons revealed a significantly lower total bacterial count in day seven with a median $6906.25 \mathrm{cfu} / \mathrm{ml}$ compared to a higher median $49887.50 \mathrm{cfu} / \mathrm{ml}$ for day 30 with an adjusted significance of 0.024. While in group II, Kruskal Wallis test showed a significant difference between the three studied time periods $(p=0.014)$. Pairwise comparisons revealed a significantly lower total bacterial count in day seven with a median $1218.75 \mathrm{cfu} / \mathrm{ml}$ compared to a median $39487.50 \mathrm{cfu} / \mathrm{ml}$ for day 30 with an adjusted significance of 0.012. As for group III, Kruskal Wallis test showed a significant difference between the three studied time periods $(p=0.003)$. Pairwise comparisons revealed also a significantly higher total bacterial count in day 30 with a median $242937.50 \mathrm{cfu} / \mathrm{ml}$ compared to a median 35831.25 $\mathrm{cfu} / \mathrm{ml}$ for day seven with an adjusted significance of 0.011 . As well as, a significantly higher total bacterial count in day 30 was noticed with a median $242937.50 \mathrm{cfu} / \mathrm{ml}$ compared to a median $30875.00 \mathrm{cfu} / \mathrm{ml}$ for day one with an adjusted significance of 0.011. (Table 2) (Figure 6)

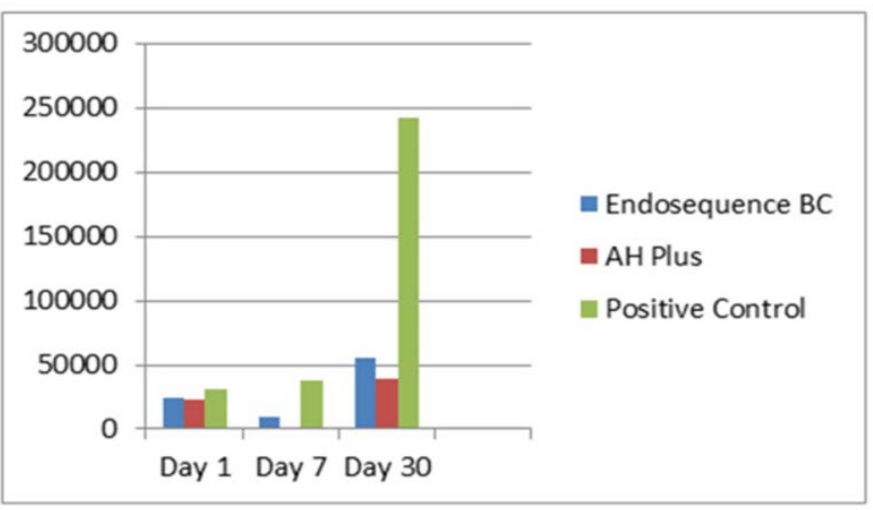

Figure (5): Total mean bacterial count at different time periods in the three studied groups.

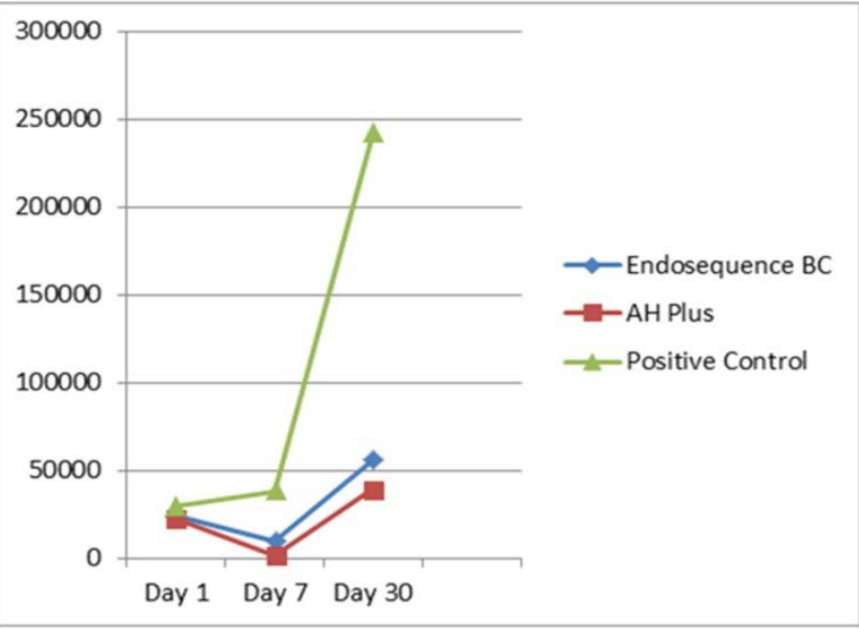

Figure (6): Total mean bacterial count changes across the studied time periods in each group.

Table (1): Showing comparison between the three studied groups regarding the total bacterial count at the different time periods. 


\begin{tabular}{|c|c|c|c|c|}
\hline $\begin{array}{l}\text { Total } \\
\text { bacterial } \\
\text { count } \\
(\mathrm{cfu} / \mathrm{ml})\end{array}$ & $\begin{array}{l}\text { Endosequence } \\
\text { BC } \\
(n=6)\end{array}$ & $\begin{array}{ll}\text { AH } & \text { Plus } \\
(\mathbf{n}=6) & \end{array}$ & $\begin{array}{l}\text { Positive } \\
\text { control } \\
(\mathrm{n}=6)\end{array}$ & $\begin{array}{l}\text { Test } \\
\text { significance } \\
p \text { (value) }\end{array}$ \\
\hline $\begin{array}{ll}\text { Day 1 } & \\
\text { Min. } & \\
\text { Max. } & \\
\text { Mean } & \pm \\
\text { SD. } & \\
\text { Median } & \\
\text { (IQR) } & \end{array}$ & $\begin{array}{l}812.50-87750.0 \\
23952.5 \pm 33127 . \\
24 \\
11488.75 \\
(2600.00- \\
29575.00)\end{array}$ & \begin{tabular}{|l|}
$162.50-$ \\
51837.50 \\
$22902.0 \pm 2022$ \\
8.2 \\
24293.50 \\
$(487.50-$ \\
$36337.50)$
\end{tabular} & \begin{tabular}{|l|}
$39487.5-$ \\
34287.5 \\
$30143.8 \pm 4193.3$ \\
30875.00 \\
$(26487.50-$ \\
$34125.00)$
\end{tabular} & $\begin{array}{l}\mathrm{X}^{2}(\mathrm{KW})(\mathrm{df}=2)=1.7 \\
66 \\
p=0.414 \mathrm{NS}\end{array}$ \\
\hline $\begin{array}{ll}\text { Day 7 } \\
\text { Min. } \\
\text { Max. } \\
\text { Mean } \quad \pm \\
\text { SD. } \\
\text { Median(IQ } \\
\text { R) }\end{array}$ & $\begin{array}{l}4712.50- \\
18200.00 \\
9614.58 \pm 5712.4 \\
4 \\
6906.25 \\
(5525.00- \\
15437.50)\end{array}$ & $\begin{array}{l}975.0-1300.0 \\
1191.7 \pm 132.7 \\
1218.75 \\
(1137.50- \\
1300.00)\end{array}$ & \begin{tabular}{|l|}
$22100.0-$ \\
63537.5 \\
$38377.1 \pm 17975$. \\
6 \\
35831.25 \\
$\quad(22262.50-$ \\
$50700.00)$
\end{tabular} & $\begin{array}{l}x \\
2\end{array}$ \\
\hline $\begin{array}{|ll|}\begin{array}{l}\text { Sig. } \\
\text { days }\end{array} & \text { bet. } \\
\end{array}$ & \multicolumn{4}{|l|}{$\mathrm{p}_{3}=0.000^{*}$} \\
\hline $\begin{array}{l}\text { Day 30 } \\
\text { Min. } \\
\text { Max. } \\
\text { Mean } \\
\text { SD. } \\
\text { Median(IQ } \\
\text { R) }\end{array}$ & \begin{tabular}{|l}
$39162.50-$ \\
92950.00 \\
$55791.67 \pm 2099$ \\
6.78 \\
49887.50 \\
$\quad(39487.50-$ \\
$63375.00)$
\end{tabular} & \begin{tabular}{|l|}
$36237.50-$ \\
43062.50 \\
$39595.83 \pm 264$ \\
5.64 \\
39487.50 \\
$(37862.50-$ \\
$41437.50)$ \\
\end{tabular} & \begin{tabular}{|l|}
$126262.5-$ \\
359287.5 \\
$243072.9 \pm 1236$ \\
63.1 \\
242937.50 \\
$(131625.0-$ \\
$355387.50)$ \\
\end{tabular} & $=0.002$ \\
\hline \begin{tabular}{|ll}
$\begin{array}{l}\text { Sig. } \\
\text { days }\end{array}$ & bet. \\
\end{tabular} & \multicolumn{3}{|c|}{$\mathrm{p}_{2}=0.048^{*}, \mathrm{p}_{3}=0.002^{*}$} & \\
\hline
\end{tabular}

n: Number of sample KS: Kolmogorov-Smirnov

Min-Max: Minimum - Maximum

*: Statistically significant $(\mathrm{p}<0.05)$

NS: Statistically not significant $(\mathrm{p} \geq 0.05)$

IQR: Inter-quartile range

KW: Kruskal-Wallis test

$\mathrm{p}_{1}$ : $\mathrm{p}$ value for comparing between Bioceramic and AH Plus $\mathrm{p}_{2}$ : $\mathrm{p}$ value for comparing between Bioceramic and Positive

\section{control}

$\mathrm{p}_{3}$ : p value for comparing between AH Plus and Positive control

Table (2): Showing comparison between the three studied periods regarding the total bacterial in each studied group.

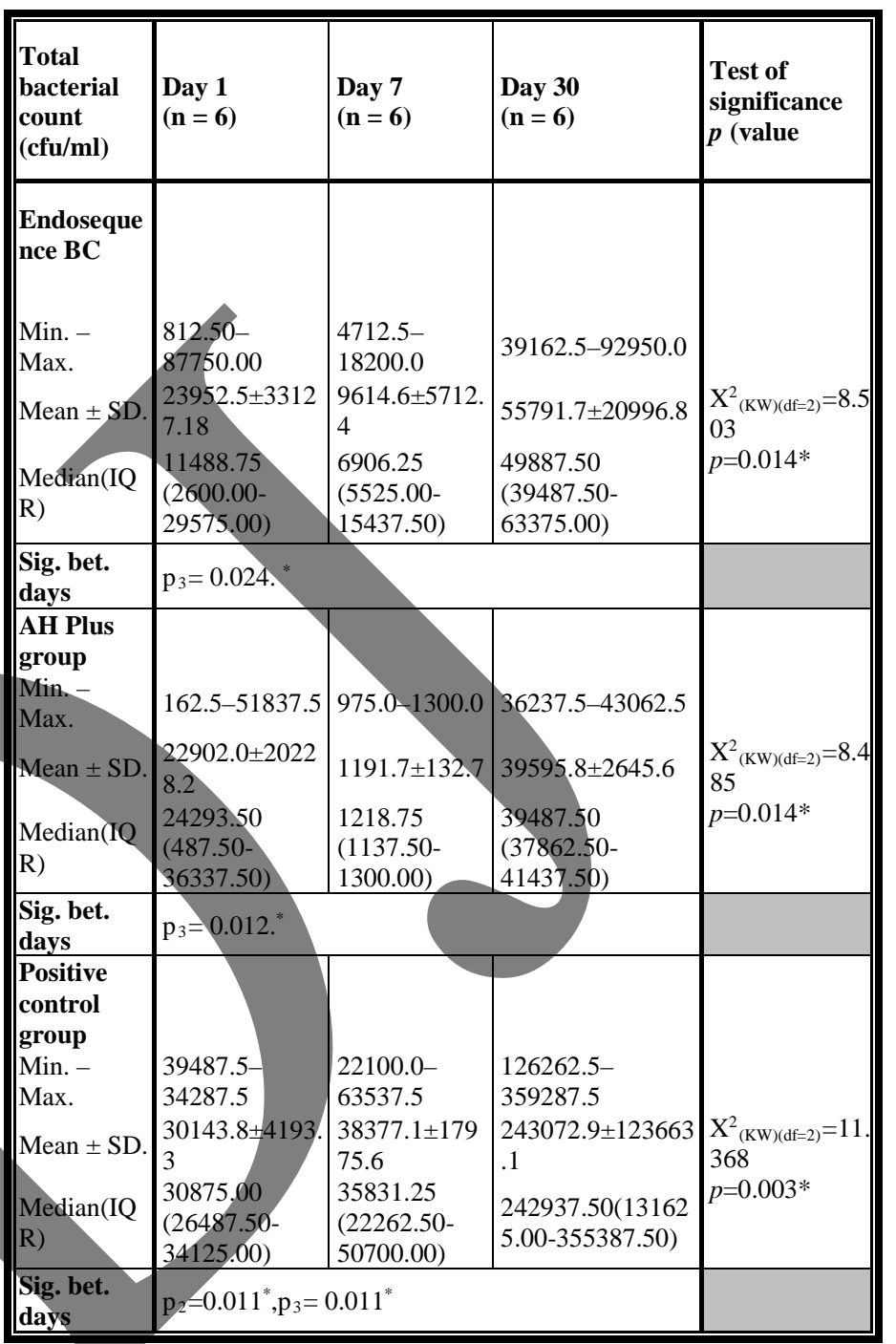

n: Number of sample

KS: Kolmogorov-Smirnov

Min-Max: Minimum - Maximum

*: Statistically significant $(\mathrm{p}<0.05)$

NS: Statistically not significant $(\mathrm{p} \geq 0.05)$

IQR: Inter-quartile range

KW: Kruskal-Wallis test

$\mathrm{p}_{1}$ : $\mathrm{p}$ value for comparing between day1 and day7

$\mathrm{p}_{2}$ : $\mathrm{p}$ value for comparing between day1 and day30

$\mathrm{p}_{3}$ : $\mathrm{p}$ value for comparing between day7 and day30

\section{DISCUSSION}

This study was conducted to study the antibacterial effect of two chemically different sealers over a 30 day period since a sealer with adequate and prolonged antibacterial effect is of the utmost importance to achieve elimination of remaining bacteria inside the root canal and thus ensure a successful treatment outcome. In the present study, the dentin block model introduced by Haapasalo and Ørstavik in the year 1987 (14) has been chosen as it has been repeatedly used in endodontic research to study the effectiveness of various irrigants, 
endodontic medicaments and root canal sealers in infected dentin against bacteria, mainly Enterococcus faecalis as it stimulates various main aspects of the in vivo conditions. Other methods of evaluation used in antimicrobial studies such as the agar diffusion test and direct contact test with its modifications lack the presence of dentin, the microanatomy and chemistry of the tooth structure as well as biofilm formation.

The dentin block was obtained from the mid root area after decoronation and apical resection, because of the presence of the wider dentinal tubule openings found in the coronal two thirds of the root thus facilitating bacterial cells penetration. The height and internal diameter were standardized among all specimens to reduce variations normally seen within the root canal system and ensure a standardized model. Cementum was grinded from the outer surface of the dentin block to standardize the size of the dentin block and to facilitate and ensure a deeper penetration of Enterococcus faecalis, with the aid of smear layer removal that was done from both intracanal and extracanal surfaces, to facilitate penetration of Enterococcus faecalis (15).

Saline was chosen as the control to demonstrate the presence of vital bacterial in the various depths examined. Following each testing time period, a microbiological culture method was employed as a sampling procedure via sequential removal of dentin from the dentin block using peeso reamers sizes three, four, five and six as it's an easy technique to perform allowing the use of a low speed handpiece to collect residual bacteria in the biofilm attached to the dentinal walls and within the dentinal tubules and confirming no cross contamination occurred with the species being studied. Live/Dead staining was avoided as it does not confirm the bacterial type within the dentinal tubules, and may lead to false results as previously suggested by $\mathrm{Ma}$ et al., (16); that the root canal contents might contain autofluoresecent and loose elements that can be confounded with bacteria, as well as background fluorescence.

In regards to assessment of the antibacterial effect with time in the Endosequence BC root canal sealer subgroups; bacterial count increase was significant between day seven and day thirty. These findings are in agreement with a study performed by Willershausen et al.,(17) which found that Endosequence BC root canal sealer exhibited no antibacterial effect when assessed by the scanning electron microscope. Furthermore, another study performed by Shin et al., (18) reported that Endosequence BC root canal sealer exhibited low antibacterial effect prior to setting and no antibacterial effect after setting.

However, for the AH Plus root canal sealer subgroups, although there was a bacterial count decrease from day one to day seven, it was not of a significant difference. However, a significant difference of bacterial count increase was noted between day seven and day 30. These results are in agreement with various studies with similar findings, that reported that $\mathrm{AH}$ Plus root canal sealer loses its antibacterial effect as it sets. Pizzo et al.,(19), reported that AH Plus exhibited antibacterial effect only when freshly mixed, this was in agreement with a study conducted by Zhang et al., (20) that showed that freshly mixed AH Plus killed Enterococcus faecalis effectively, but for one-day and three-day samples, no significant antibacterial activity was noted. Furthermore, Prasad (21) found that the percentage of inhibition decreased with the increase in settling time, seven days showed the least antibacterial effect. However, this was in contrast with findings of Wang et al., (22), as well as Du et al., (23) who reported that antibacterial activity of $\mathrm{AH}$ Plus root canal sealer lasted up to 30 days after setting. This might be related to biased results, as both papers were published by the same authors, or due to different experimental designs (i.e non standardization of antibacterial testing methods).

These findings that illustrate that both sealers lose their antimicrobial effect with time are in agreement with other studies conducted; Gomes et al., (24) illustrated that AH plus sealer did not totally inhibit the microbial growth, and its antimicrobial activity decreased with time. This was in agreement with Shakouie et al., (25). Furthermore, Ustun et al., (26) found that fresh iRoot SP (a Ca2SiO4 root canal sealer) and fresh AH Plus had bactericidal action against E. faecalis but decreased with time to become bacteriostatic.

The bacterial count increase over the thirty day period in the Endosequence BC group might be attributed to the dentin buffering efffect. A study conducted by McHugh et al., (27) illustrated that Enterococcus faecalis is unable to survive in a $\mathrm{pH}$ of 11.5 or greater, although the manufacturer states that Endosequence BC root canal sealer exhibits a high antibacterial effect due to a $+12 \mathrm{pH}$ upon setting, the dentin's buffering effect may mask that effect and make it difficult for the sealer to maintain such a high $\mathrm{pH}$ (28). This theory is supported by a study conducted by Wang and Hume (29) that illustrated that dentin exhibited strong buffering capacity against acids with considerable buffering capacity against alkali.

Eurthermore, the proton pump of Enterococcus faecalis aids in the resistance to alkali and thus increases its survival after obturation with calcium silicate based sealers. This of course contradicts the results found by Wang et al., (22) that illustrate that dentin prolongs the antibacterial ability of endodontic sealers such as Endosequence $\mathrm{BC}$ and $\mathrm{AH}$ plus against Enterococcus faecalis biofilms in the dentinal tubular system. The authors claimed that this long term action may be due to the antibacterial compounds from the sealers interacting with released contributing factors from dentin such as silica.

As for the positive control subgroups, significant bacterial count increase was noted throughout the studied time periods. This is of course was due to lack of sealer presence and thus the absence of any antibacterial effect.

When the three main groups were compared after one day of incubation in the present study, no significant difference in the total bacterial count among the three groups was noted, while after seven days a significantly lower total bacterial count in the AH plus root canal sealer group was noted compared to the positive control group. This highlights deeper antimicrobial action over time for the AH Plus root canal sealer when compared to the one day incubation period. This may be the 
result of the relative insolubility of $\mathrm{AH}$ plus as previously highlighted by Urban et al., (30) this insolubility may lead to bacterial entombing and thus reduces its chances of survival.

Leonardo et al., (31) illustrated that AH Plus released bisphenol A diglycidyl ether and formaldehyde during polymerization which may be a sharing factor in its antibacterial activity. However, in this study after 30 days of incubation both $\mathrm{AH}$ Plus and Endosequence BC root canal sealers showed an indicatively lower total bacterial count compared to the positive control group. According to the manufacturer Endosequence BC is chemically composed of $\mathrm{Ca} 2 \mathrm{SiO} 4$, calcium phosphate and calcium oxide. In the present study Endosequence BC sealer managed to maintain an antibacterial effect in comparison to the positive control group throughout the 30 day period. Zhang et al., (5) claimed that the prolonged antibacterial ability of Endosequence BC may be due to the biomineralization process happening as a result of the calcium silicates and phosphates present in the the sealer's components along with the dentine mineral participation. $\mathrm{PH}$ is elevated as a result of a continuous hydration reaction from the dentine's moisture that produces a hydrogel of $\mathrm{Ca} 2 \mathrm{SiO} 4$ as well as $\mathrm{Ca}(\mathrm{OH}) 2$. A study by Zehnder et al., (32) illustrated that the dissolving action of Silica an alkaline environment may directly inhibit bacterial viability. Moreover, Calcium hydroxide in turn reacts with the phosphate to form hydroxyapatite and water. According to Yang et al., (33) the resultant water is supposed to participate in the reaction cycle once more to produce more hydrogel of $\mathrm{Ca} 2 \mathrm{SiO} 4$ and more $\mathrm{Ca}(\mathrm{OH}) 2$. Thus, the ongoing diffusion of calcium hydroxide into the dentinal tubular system may explain the continuous killing of bacteria throughout the 30-day period.

Furthermore, a study conducted by Kapralos et al., (3) is in agreement with the present study's findings, they found that AH Plus exhibits a stronger antibacterial activity against biofilms of Enterococcus faecalis when compared with a Ca2SiO4 based sealer (Totalfill BC sealer). However, a limitation of this study is that it was conducted on a single bacterial species, not stimulating true endodontic infections, thus assessment of the antibacterial effect of both sealers on poly-microbial infections and on dentinal tubule disinfection is recommended in future in-vitro studies.

\section{CONCLUSIONS}

In conclusion, the null hypothesis was rejected since AH plus exhibited a stronger antibacterial affect after setting. Therefore, it can be concluded that $\mathrm{AH}$ Plus was more effective than Endosequence BC in eliminating Enterococcus faecalis from the root canal system. In addition, both sealers lost their antibacterial effect with time.

\section{CONFLICT OF INTREST}

The authors declare that they have no conflicts of interest.

\section{REFERENCES}

1. Spångberg LS, Haapasalo M. Rationale and efficacy of root canal medicaments and root filling materials with emphasis on treatment outcome. Endod Topics. 2002;2:35-58.

2. Stuart $\mathrm{CH}$, Schwartz SA, Beeson TJ, Owatz CB. Enterococcus faecalis: its role in root canal treatment failure and current concepts in retreatment. J Endod. 2006;32:93-8.

3. Kapralos V, Koutroulis A, Ørstavik D, Sunde PT, Rukke HV. Antibacterial activity of endodontic sealers against planktonic bacteria and bacteria in biofilms. J Endod. 2018;44:149-54.

4. Zhou HM, Shen Y, Zheng W, Li L, Zheng YF, Haapasalo M. Physical properties of 5 root canal sealers. J Endod. 2013;39:1281-6.

5. Zhang H, Shen Y, Ruse ND, Haapasalo M. Antibacterial activity of endodontic sealers by modified direct contact test against Enterococcus faecalis. J Endod. 2009;35:1051-5.

6. Saleh IM, Ruyter IE, Haapasalo M, Ørstavik D. Survival of Enterococcus faecalis in infected dentinal tubules after root canal filling with different root canal sealers in vitro. Int Endod J. 2004;37:193-8.

7. Vivacqua-Gomes N, Gurgel-Filho ED, Gomes BP, Ferraz CC, Zaia AA, Souza-Filho EJ. Recovery of Enterococcus faecalis after single-or multiple-visit root canal treatments carried out in infected teeth ex vivo. Int Endod J. 2005;38:697-704.

8. Heling I, Chandler NP. The antimicrobial effect within dentinal tubules of four root canal sealers. J Endod. 1996;22:257-9.

9. I Spss I. IBM SPSS/statistics version 21. Boston, Mass: IBM. Corp. 2012;126.

10. Field A. Discovering statistics using IBM SPSS statistics. sage; 2013.

11. Snecdecor GW, Cochran WG. Stat. Methods: Wiley; 1991.

12. Kruskal WH, Wallis WA. Use of ranks in one-criterion variance analysis. J. Am. Stat. Assoc. 1952;47:583-621.

13.Dunn OJ. Multiple comparisons using rank sums. Technometrics. 1964;6:241-52.

14. Haapasalo M, Ørstavik D. In vitro infection and of dentinal tubules. J Dent Res. 1987;66:1375-9.

15. Alyahya A, Alqareer A. Does the Removal of Cementum Facilitate Bacterial Penetration into Dentinal Tubules In Vitro?. J Endod. 2017;43:1111-5.

16. Ma J, Wang Z, Shen Y, Haapasalo M. A new noninvasive model to study the effectiveness of dentin disinfection by using confocal laser scanning microscopy. J Endod. 2011;37:1380-5.

17. Willershausen I, Callaway A, Briseño B, Willershausen B. In vitro analysis of the cytotoxicity and the antimicrobial effect of four endodontic sealers. Head Face Med. 2011;7:15.

18. Shin JH, Lee DY, Lee SH. Comparison of antimicrobial activity of traditional and new developed root sealers against pathogens related root canal. J Dent Sci. 2018;13:54-9.

19. Pizzo G, Giammanco GM, Cumbo E, Nicolosi G, Gallina G. In vitro antibacterial activity of endodontic sealers. J Dent. 2006;34:35-40. 
20.Zhang H, Pappen FG, Haapasalo M. Dentin enhances the antibacterial effect of mineral trioxide aggregate and bioaggregate. J Endod. 2009;35:221-4.

21. Prasad MP. In-vitro antimicrobial evaluation of Endodontic cavity sealers against Enterococcus faecalis. IJIRSET. 2013;2:7662-6.

22. Wang Z, Shen Y, Haapasalo M. Dentin extends the antibacterial effect of endodontic sealers against Enterococcus faecalis biofilms. J Endod. 2014;40:505-8.

23. Du T, Wang Z, Shen Y, Ma J, Cao Y, Haapasalo M. Combined antibacterial effect of sodium hypochlorite and root canal sealers against Enterococcus faecalis biofilms in dentin canals. J Endod. 2015;41:1294-8.

24. Gomes BP, Pedroso JA, Jacinto RC, Vianna ME, Ferraz CC, Zaia AA, et al. In vitro evaluation of the antimicrobial activity of five root canal sealers. Braz Dent J. 2004;15:30-5.

25. Shakouie S, Esk M, Shahi S, FroughReihani M, Soroush M, Gosili A. Antimicrobial efficacy of AH-Plus, adseal and endofill against Enterococcus faecalis-An in vitro study. Afr J Microbiol Res. 2012;6:991-4..

26. Ustun Y, Sagsen B, Durmaz S, Percin D. In vitro antimicrobial efficiency of different root canal sealers against Enterecoccus faecalis. European J Gen Dent. 2013;2:134.
27. McHugh CP, Zhang P, Michalek S, Eleazer PD. pH required to kill Enterococcus faecalis in vitro. J Endod. 2004;30:2189.

28. Haapasalo M, Qian W, Portenier I, Waltimo T. Effects of dentin on the antimicrobial properties of endodontic medicaments. J Endod. 2007;33:917-25.

29. Wang JD, Hume WR. Diffusion of hydrogen ion and hydroxyl ion from various sources through dentine. Int Endod J. 1988;21:17-26.

30. Urban K, Neuhaus J, Donnermeyer D, Schäfer E, Dammaschke T. Solubility and $\mathrm{pH}$ value of 3 different root canal sealers: a long-term investigation. $\mathrm{J}$ Endod. 2018;44:1736-40.

31. Leonardo MR, da Silva LA, Tanomaru Filho M, da Silva RS. Release of formaldehyde by 4 endodontic sealers. Oral Surg Oral Med Oral Pathol Oral Radiol Endod. 1999;88:221-5.

32.Zehnder M, Waltimo T, Sener B, Söderling E. Dentin enhances the effectiveness of bioactive glass S53P4 against a strain of Enterococcus faecalis. Oral Surg Oral Med Oral Pathol Oral Radiol Endod. 2006;101:530-5.

33. Yang Q, Troczynski T, Liu DM. Influence of apatite seeds on the synthesis of calcium phosphate cement. Biomaterials. 2002;23:2751-60

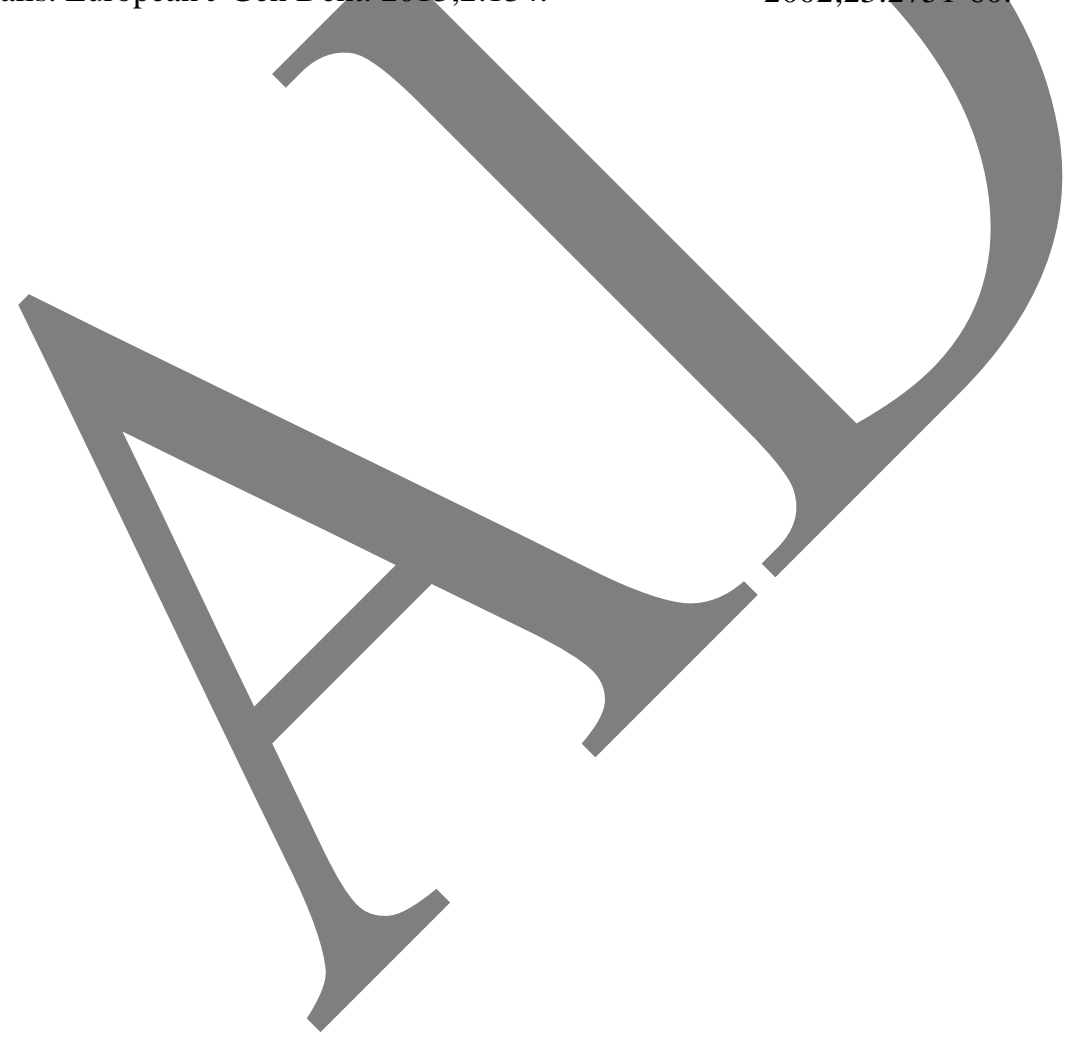

